\title{
Correction to: Shoot regeneration process and optimization of Agrobacterium-mediated transformation in Sinningia speciosa
}

\author{
Wen-Hsi Kuo ${ }^{1} \cdot$ Yu-Ling Hung ${ }^{2,3} \cdot$ Ho-Wei Wu ${ }^{2,4} \cdot$ Zhao-Jun Pan $^{5} \cdot$ Chwan-Yang Hong ${ }^{6} \cdot$ Chun-Neng Wang ${ }^{5}$
}

Published online: 27 June 2018

(c) Springer Nature B.V. 2018

\section{Correction to: \\ Plant Cell, Tissue and Organ Culture (PCTOC) https://doi.org/10.1007/s11240-018-1424-7}

All hormone and antibiotic concentrations listed in the original publication were incorrect as $\mathrm{mg} / \mathrm{mL}$. They should be corrected as "mg/L".

In the first paragraph of the Results section "Histological observation of plant regeneration", the best shooting condition was not clear as $(0.1$ and $1.0 \mathrm{mg} / \mathrm{mL} \mathrm{BA})$. The best shooting condition was in fact as "( $0.1 \mathrm{mg} / \mathrm{L}$ NAA and $1.0 \mathrm{mg} / \mathrm{L}$ BA)".

In the Materials and methods section "Agrobacteriummediated genetic transformation", the $\mathrm{pH}$ values of the inoculation medium and co-culture medium were not mentioned. They should be adjusted to pH 5.2 before autoclave sterilization.

The authors apologize for these errors.
The original article can be found online at https://doi.org/10.1007/ s11240-018-1424-7.

Chun-Neng Wang

leafy@ntu.edu.tw

Wen-Hsi Kuo

kuowenhsi@gmail.com

Yu-Ling Hung

yuling7881@gmail.com

Ho-Wei Wu

b99b01053@ntu.edu.tw

Zhao-Jun Pan

zhaojunpan@ntu.edu.tw

Chwan-Yang Hong

cyhong@ntu.edu.tw

1 Institute of Ecology and Evolutionary Biology, National

Taiwan University, No. 1, Sec. 4, Roosevelt Rd.,

Taipei 10617, Taiwan, Republic of China
2 Department of Life Science, National Taiwan University, No. 1, Sec. 4, Roosevelt Rd., Taipei 10617, Taiwan, Republic of China

3 Present Address: Institute of Plant Biology, National Taiwan University, No. 1, Sec. 4, Roosevelt Rd., Taipei 10617, Taiwan, Republic of China

4 Present Address: Institute of Plant and Microbial Biology, Academia Sinica, No. 128, Sec. 2, Academia Rd., Taipei, Taiwan, Republic of China

5 Department of Life Science, Institute of Ecology and Evolutionary Biology, National Taiwan University, No. 1, Sec. 4, Roosevelt Rd., Taipei 10617, Taiwan, Republic of China

6 Department of Agricultural Chemistry, National Taiwan University, No.1, Sec. 4, Roosevelt Rd., Taipei 10617, Taiwan, Republic of China 